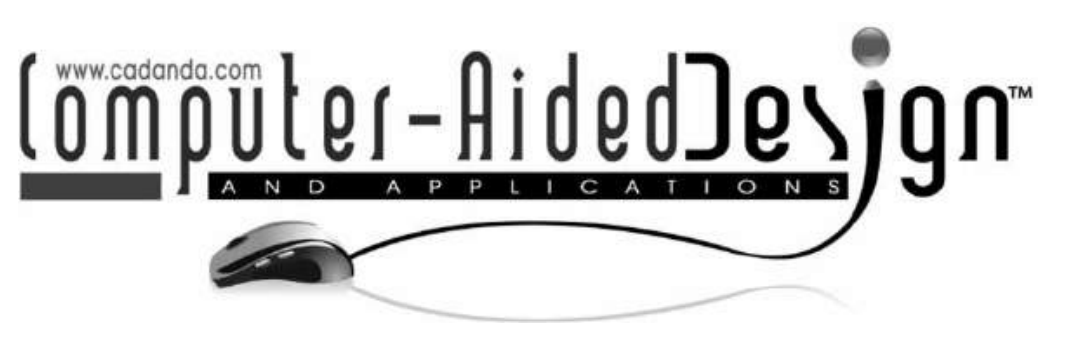

\title{
Simulation Methods in the Foot Orthosis Development Process
}

\author{
Samuel J. Lochner, Jan P. Huissoon, Sanjeev S. Bedi \\ University of Waterloo, sjlochne@engmail.uwaterloo.ca
}

\begin{abstract}
Traditional methods for developing foot orthoses require extensive skilled manual labor. More modern methods have sought to address this with the introduction of computer enabled technologies such as digital scanning, computer aided design, and automated manufacturing. The current work further advances the process with the introduction of an additional computer enabled technology, simulation models, into two additional steps. First, a simulation model is used to achieve the postural adjustments to the foot normally done by a practitioner. This has the benefit of further automating the process, improving repeatability, and preventing the deformation of the plantar soft tissues that normally occurs with physical postural adjustment. Second, the simulation model is used in a routine to optimize plantar pressure distribution. When compared to a conventional method, the proposed approach yielded a $61 \%$ reduction in peak plantar pressure. Future work includes automating the optimization routines for a variety of metrics. Other applications for the current work include the development processes of orthoses and prostheses for other parts of the body.
\end{abstract}

Keywords: foot orthosis, finite element, simulation, optimization.

\section{INTRODUCTION}

A foot orthoses (FO) is intended to prevent injury or aid in recovery by acting to redistribute pressure experienced by the plantar surface of the foot and/or cause adjustments to relative bone positions during standing and gait. FO geometry originates from a duplicate of the plantar foot surface and then geometrical deviations are made to this base shape in order to control how the FO influences plantar pressure and foot posture. Methods for developing FOs can be categorized as either traditional or modern, where traditional methods rely on manual techniques and modern methods include new computer enabled technologies.

\subsection{Traditional FO Development Methods}

A variety of paradigms for controlling posture exist, the earliest being that proposed by Merton Root in the 1970's [22]. While Root's methods have been questioned or criticized [15,23], they still form the predominant theory known and practiced today. Though the current work could be adapted to a variety of different postural control paradigms, it will be adapted here to Root's approach. Traditional methods following Root's techniques and those later described in greater detail by J.W. Philps in, "The Functional FO" [20] can be summarized and broken down into the following five steps:

1. Patient assessing: The practitioner examines the patient while non weight bearing and during standing and gait. Any pathologies are noted and appropriate orthotic interventions recommended. The tibial stance, subtalar joint, and midtarsal joint angles are measured with the subtalar joint in the neutral position and the midtarsal joint locked to arrive at the ideal midstance rearfoot and forefoot angles. Various characteristics of the FO are decided at this point such as the FO's type, materials, and any local regions requiring decreased pressure. The definitions for the angular measurements are as follows:

- The tibial stance angle is measured in the frontal plane as the angle between a line bisecting the lower leg and vertical during standing.

- The subtalar joint angle is measured in the frontal plane as the angle between the lower leg bisection line and a line bisecting the calcaneus. 
- The midtarsal joint angle is measured in the frontal plane as the angle between a line connecting the metatarsal joints along the plantar surface of the foot and the calcaneus bisection line.

- The rearfoot angle is the addition of the tibial stance angle and the subtalar joint angle. Or, more simply, it is the angle between the calcaneal bisection and the sagital plane as viewed in the frontal plane.

- The forefoot angle is the addition of the rearfoot angle and the midtarsal joint angle. Or, more simply, it is the angle between a line connecting the metatarsophalangeal joints along the plantar surface of the foot and the transverse plane as viewed in the frontal plane.

2. Foot geometry recording: The practitioner casts the foot while maintaining the subtalar joint neutral position and locked midtarsal joint as well as neutral ankle position where the ankle is neither plantar flexed nor dorsiflexed. The patient may be either supine or prone. A positive cast is created by pouring Plaster of Paris into the negative cast.

3. FO geometry developing: A mould for developing the FO geometry is formed by modifying the positive cast. Modifications are necessary in order to ensure the FO formed about the cast will achieve the pressure adjustments and postural adjustments (intrinsic posting) prescribed by the practitioner and are achieved through the addition and subtraction of plaster.

4. FO fabricating: At this stage in the process, the manner in which the FO controls posture has already been determined through the preceding steps. The goal of this step is to fabricate a FO with a top surface that matches the altered positive cast from step 3 and attach support material to the bottom of the FO (posting) to ensure it remains oriented in the shoe as prescribed in step 1. Vacuum forming is used to create the FO components about the plantar foot surface.

5. FO fitting and adjusting: Some adjustments may be necessary in order to fit the FO into the intended shoe and to adjust the FO's ability to control posture and pressure.

\subsection{Modern FO Development Methods}

From the above steps, it is clear that traditional methods require an extensive amount of skilled manual labor. This has implications for both cost and repeatability. Modern FO development methods stand to make improvements through the adoption of computer enabled technologies such as digitizers, computer aided design (CAD), and automated manufacturing. It was during the mid 1980s that researchers began to publish on the use of computer enabled advancements for the application of orthosis and prosthesis development $[5,13]$ and more specifically, FO development [11]. Since then, researchers have published on the continuing advancements in digitizing [14,21], designing [10], optimizing [1,9,25], fabricating $[19,24]$, as well as advancements to overall procedures $[3,8]$ and finite element $(\mathrm{FE})$ investigations of FO design parameters $[2,4,6,7,12,26]$.

Steps 2 through 4 of the FO development process have been most influenced by technological advancement. In step 2, the foot geometry is captured as a digital rather than physical entity with various types of scanners. Though these scanners produce geometrically accurate representations of the outer surface of the foot, the mechanics of the foot are disregarded given the static nature of the models. In step 3, CAD software is used to develop the FO geometry or a mould about which the FO will be fabricated. The full potential of CAD, including iterative optimization routines, has yet to be realized. In step 4, various automated manufacturing methods are used to fabricate the FO. Though these automated fabrication methods are more efficient than traditional manual methods, FO fitting and adjusting remains a time consuming step. The proposed method aims to reduce dependence on fitting and adjusting through the adoption of advanced simulation methods.

\subsection{Proposed FO Development Method}

The proposed solution makes use of an additional computer enabled technology, simulation models, in two additional steps. The first use of the simulation models is to carry out all postural adjustments. This has the benefit of further automating the process, improving repeatability, and preventing the distortion of the plantar soft tissues that normally occurs with physical postural adjustment. The second role of the simulation model is to guide an iterative FO design approach; the foot is loaded onto the FO and some metric (plantar pressure in the example below) is calculated and the design process is repeated until the metric reaches an acceptable value. Fig. 1 depicts both the conventional steps for producing a FO and the proposed method.

A simulation method is required that can be used to model postural adjustments and loading of a foot on a FO. Two common types of models used for simulation of foot mechanics are the multi-body model and the FE model. Though the computational efficiency of the multi-body model would be beneficial to the current application, it lacks the FE method's ability to model the tissue deformation that occurs as a foot becomes weight bearing. Hence, the FE approach was implemented for the current research. FE foot models have become common medical and engineering tools in recent years. Its application, however, has primarily been limited to research as few clinical applications 


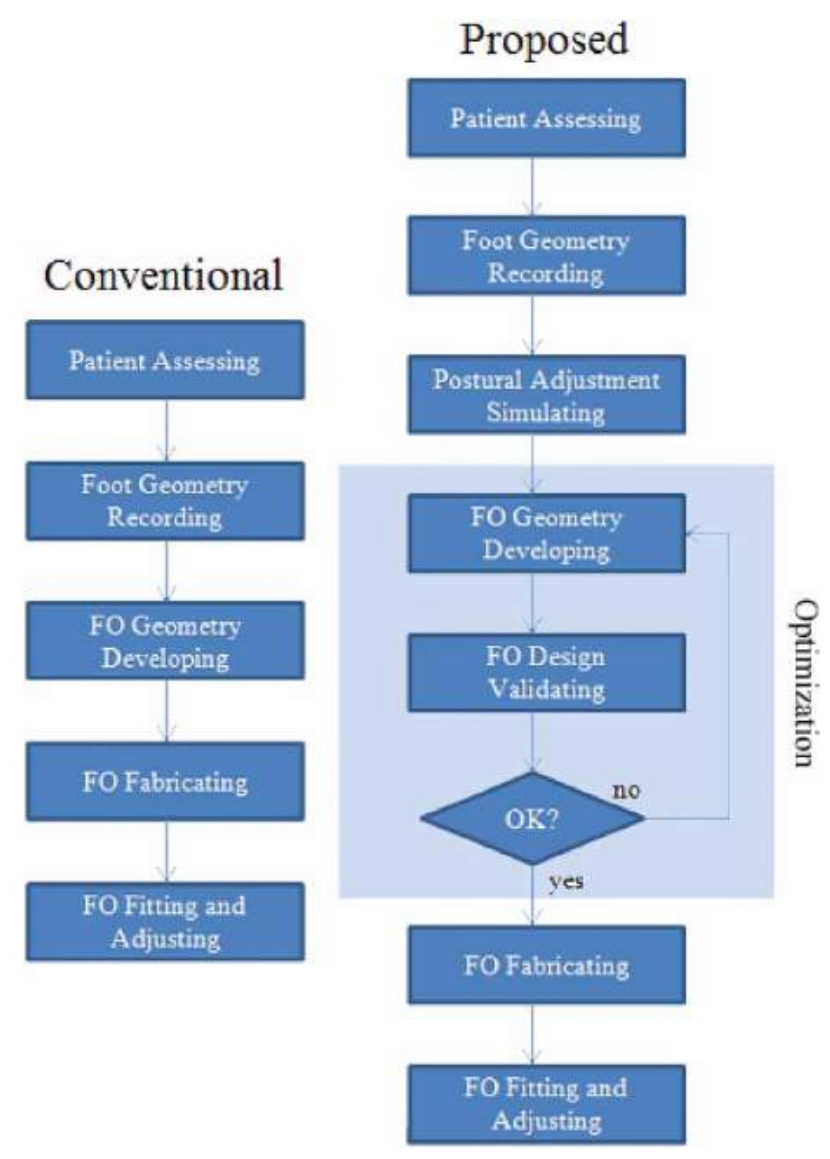

Fig. 1: Flow charts for conventional FO development methods and the proposed method which incorporates simulation techniques and a pre-fabrication optimization routine.

warrant the development cost of a FE foot model. High cost stems from the MRI or CT scan and the skilled labour required to assemble the model for FE analysis. Consequently, the FE modelling approach has previously been out of reach for the application of FO development. The solution employed for the current work is to deform a detailed generic FE foot model to an inexpensive surface scan obtained from a modern digitizer in order to develop a patient-specific FE foot model. The details of this procedure can be found in the authors' previous works $[17,18]$. It is not the purpose of this current work to elaborate on or validate this method of rapidly developing a simulation model, but rather to demonstrate and discuss its incorporation, or the incorporation of any other simulation model for that matter, into a novel process for developing a FO.

\section{METHODS}

The 7 steps of the proposed process were carried out for a single subject as follows:
1. Patient assessing: The tibial stance angle was measured during weight bearing and the subtalar and midtarsal joint angles were measured with the patient prone and the foot in the subtalar neutral position with the midtarsal joint locked. The resulting angles were $5.7^{\circ}$ valgus, $2.1^{\circ}$ varus, and $6.6^{\circ}$ varus yielding a rearfoot angle of $3.6^{\circ}$ valgus and a forefoot angle of $3.0^{\circ}$ varus. Other postural adjustments decided upon at this point were positioning the foot for a $10 \mathrm{~mm}$ heel height and for a toe spring with a $100 \mathrm{~mm}$ arc radius to demonstrate how the foot posture can be adjusted to match intended footwear.

2. Foot geometry recording: An Artec 3D handheld scanner (MH, San Jose, USA) was used to capture the foot geometry and landmarks while the foot was non weight bearing and relaxed with the subject prone.

3. Postural adjustment simulating: The generic FE foot model developed by Lochner [18] from MRI data was morphed to the subject's foot with the method developed by Lochner et al. [17] in order to form the patient specific FE foot model (Fig. 2). In the FE software, Abaqus (Waltham, USA), boundary conditions were applied to the bones to achieve the posture prescribed in step 1 as follows (Fig. 3, Fig. 4, Fig. 5):

a. The foot was fully constrained in all DOF at the ankle (tibia and fibula).

b. Points on the inferior surface of the distal heads of the first and fifth metatarsals were displaced along the Z-axis in order to achieve a heel height of $10 \mathrm{~mm}$ and a forefoot angle of $3.0^{\circ}$ varus.

c. A point on the posterior/inferior surface of the calcaneus was displaced along the Y-axis in order to achieve a rearfoot angle of $3.6^{\circ}$ valgus.

d. A point on the anterior surface of the first toe was displaced along the Z-axis in order to achieve a toe spring with a $100 \mathrm{~mm}$ radius.

e. A point on the inferior surface of the intermediate cuneiform can be displaced vertically in order to increase arch height of the resulting FO. This adjustment was not used as the current subject had a high arch and did not need any further support in the arch area.

4. FO geometry developing: The FO was designed about the posturally adjusted foot from step 3 using the FO design program developed by Lochner et al. [16] (Fig. 6). Ordinarily, in this step the complete foot model including extrinsic posts and other features is developed and prepared for fabrication. For the current purposes, only a rigid uniform thickness part was designed for analysis purposes in the next step. 


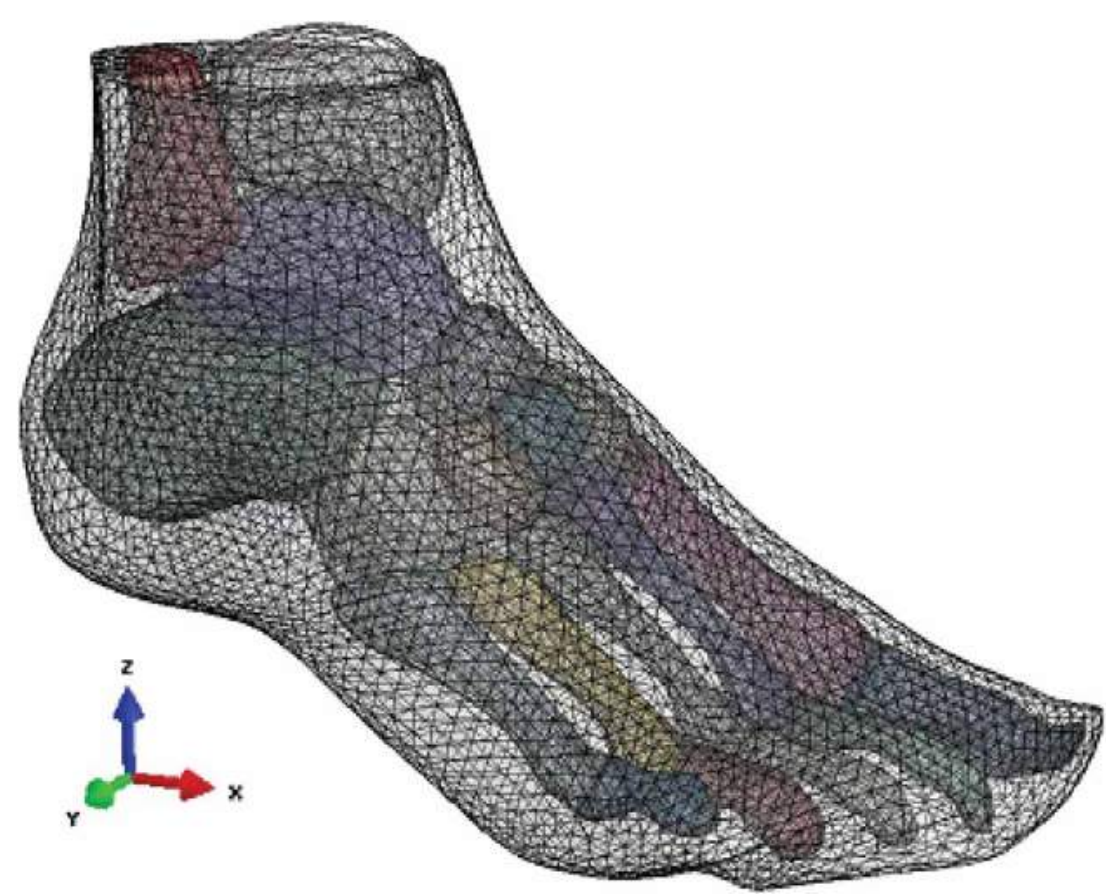

Fig. 2: The meshed FE model that has been morphed to the subject's foot.

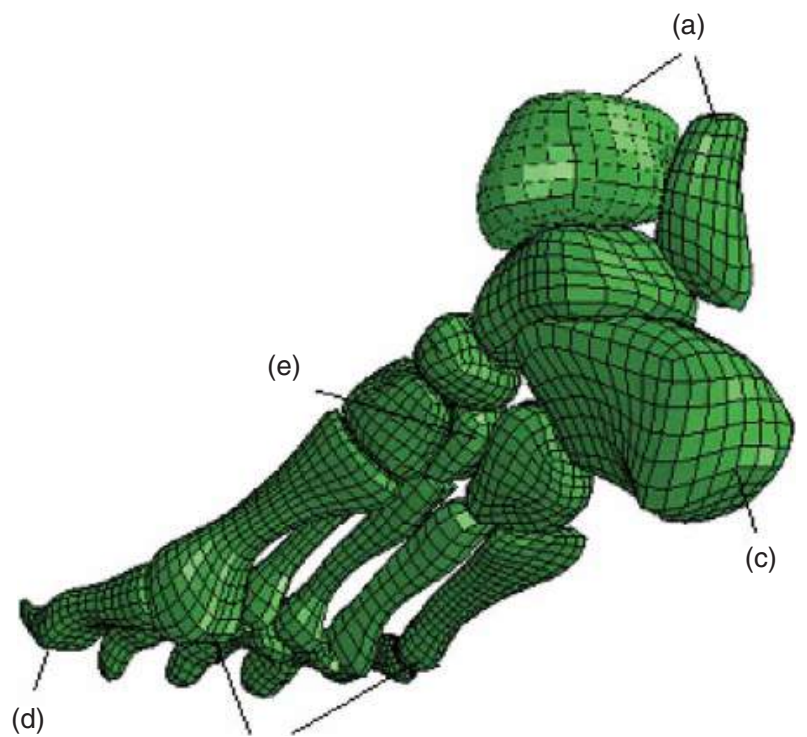

(b)

Fig. 3: Locations of boundary conditions for adjusting the foot's posture: a) tibia and fibula, b) first and fifth metatarsal heads, c) calcaneus, d) first toe, e) intermediate cuneiform.

5. FO design validating: A vertical force was applied to the FO (half of subject's weight) and a second vertical force was applied to the calcaneus where the Achilles tendon attaches $(65 \%$ of the first force in order to achieve a center of pressure similar to that from pressure mat data) (Fig. 7). Boundary conditions were

applied to the foot and FO to restrict movement during loading (Fig. 7). A displacement step was used to achieve contact between the foot and FO followed by a loading step to simulate weight bearing force. Fig. 8 depicts the resulting simulated plantar pressure distribution. The control points for the FO surface were vertically displaced by $1 \mathrm{~mm}$ increments until pressures in peak pressure regions were reduced to the levels of surrounding regions (Fig. 8).
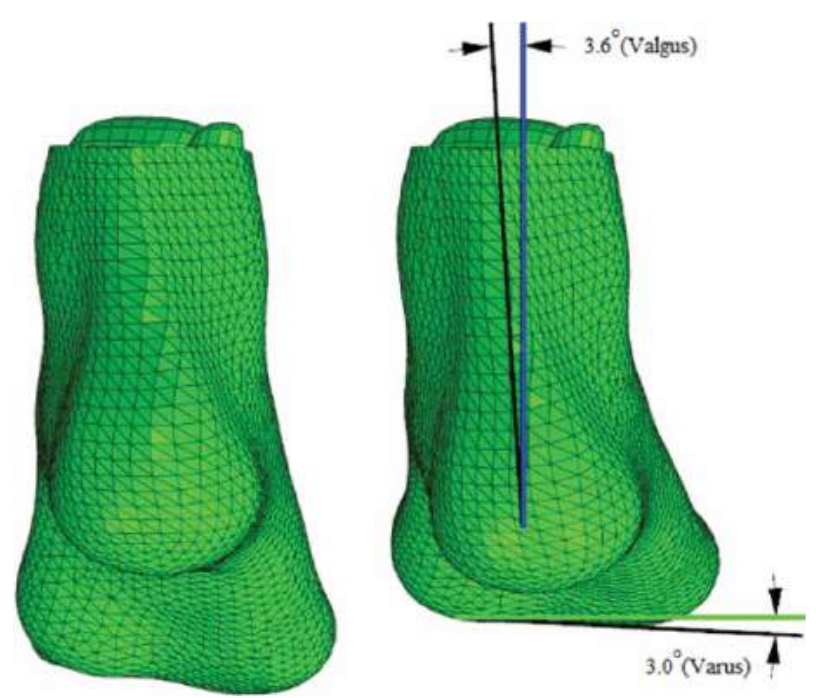

Fig. 4: Rearfoot and forefoot angles in the frontal plane before and after postural adjustment. 

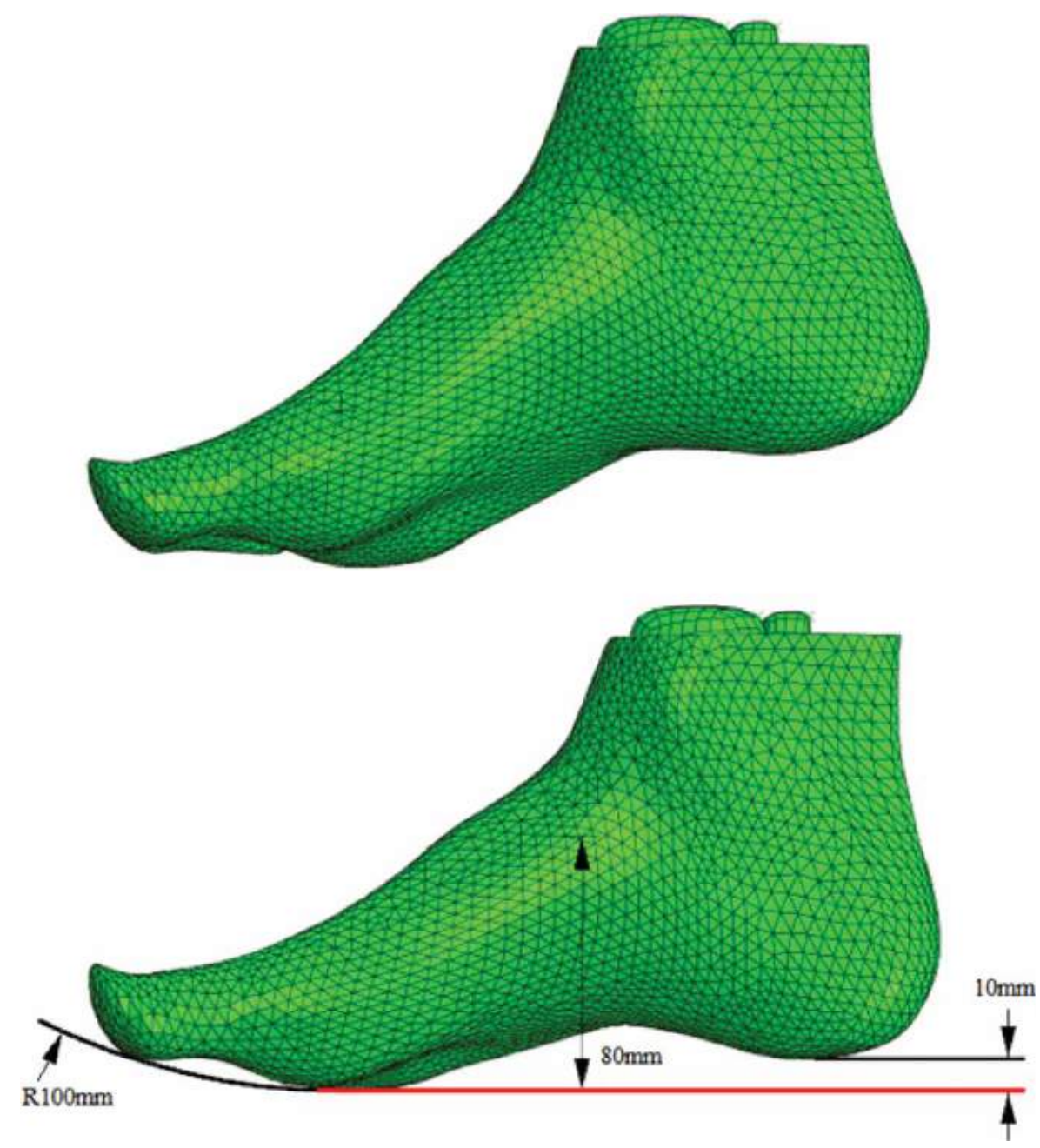

Fig. 5: Heel height, arch height, and toe spring in the sagital plane before and after postural adjustment.
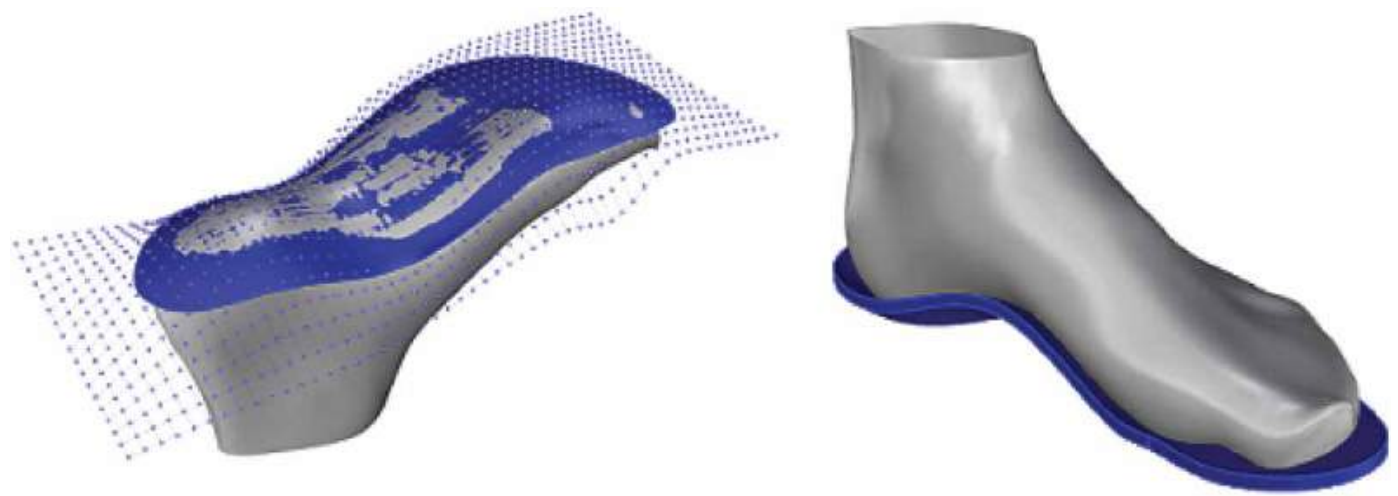

Fig. 6: The FO top surface after being designed about the plantar surface of the foot.

6. FO fabricating: The next step in the process is to fabricate the FO. Any technique may be used however additive manufacturing is most suited to the goal of reducing skilled manual labor as the complete 3D FO design can be created without manual processes.

7. FO fitting and adjusting: This step remains unchanged from the conventional process. 


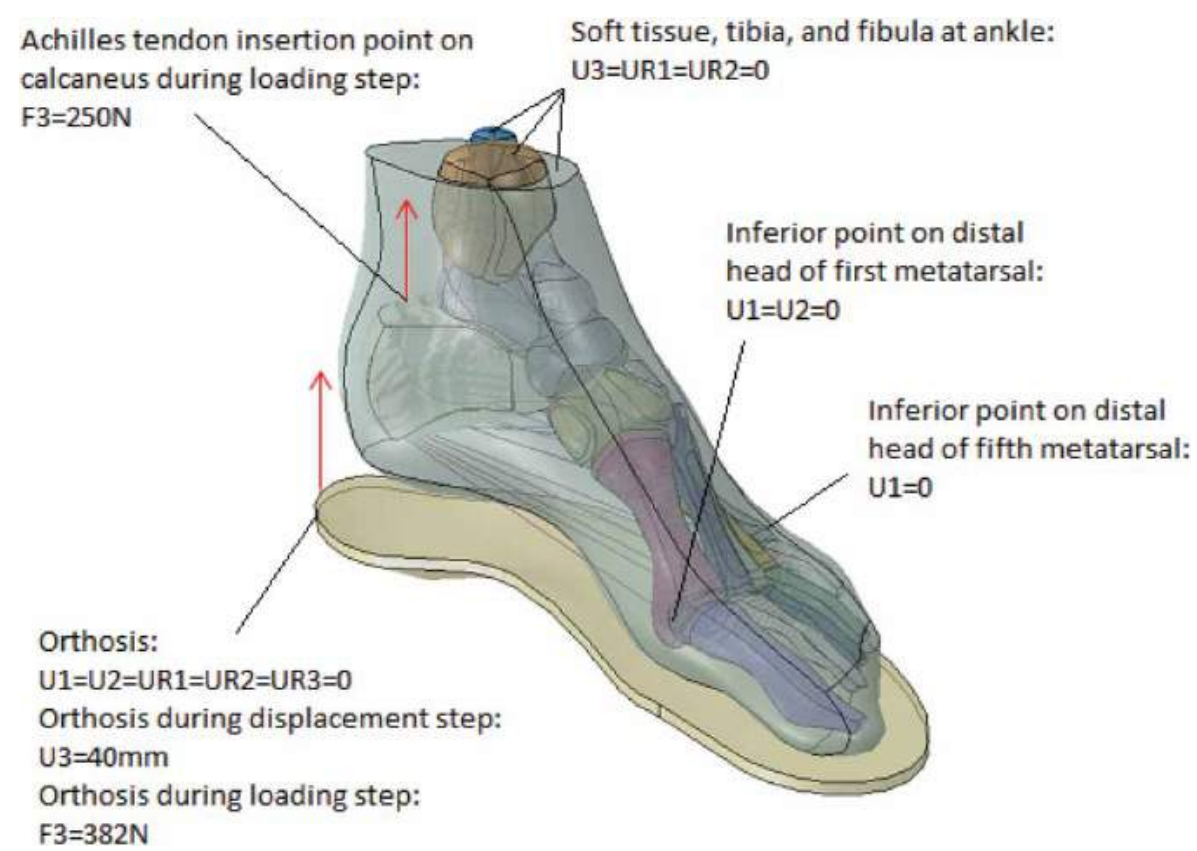

Fig. 7: Boundary conditions and loads for simulating weight bearing on the FO.

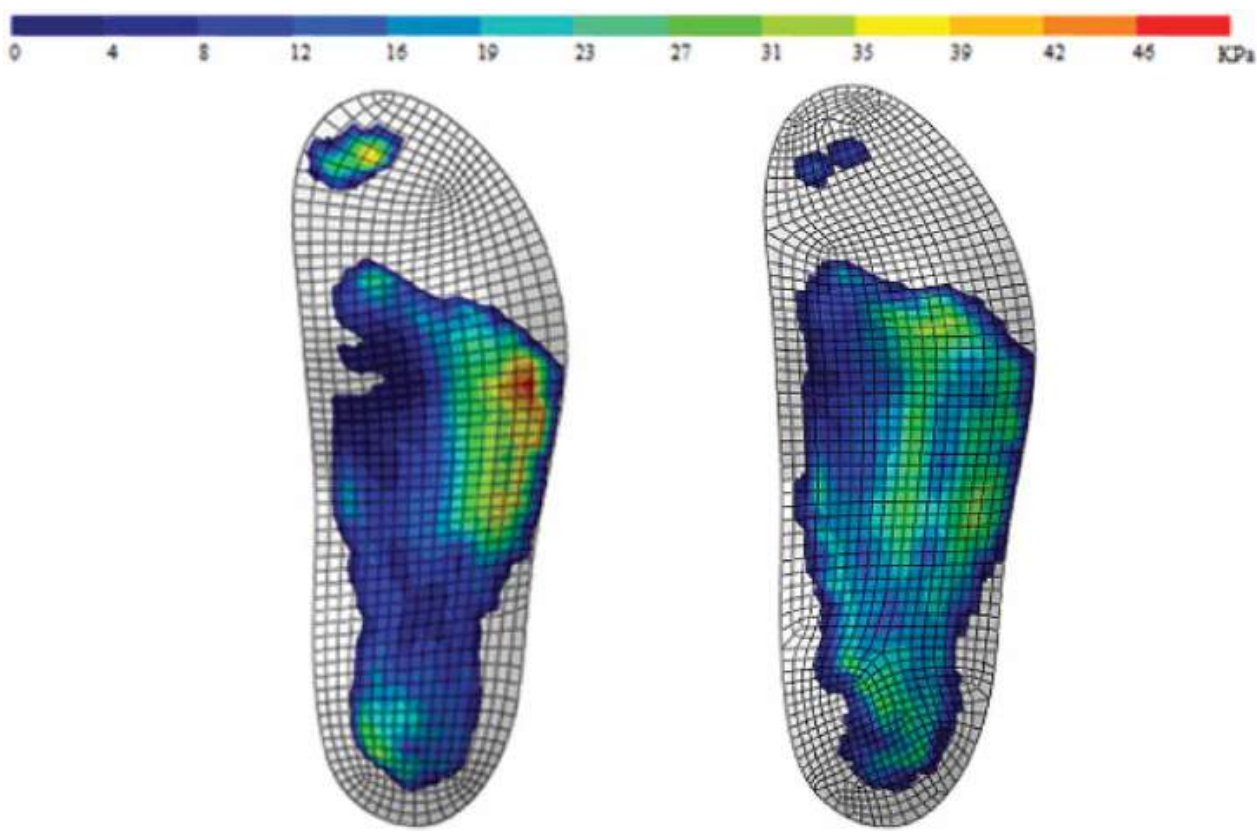

Fig. 8: Pressure distributions yielded by initial and optimized FOs.

However, reliance on this step is diminished due to the addition of the optimization routine prior to fabrication thereby improving efficiency and reducing the overall cost of the process.

To demonstrate the benefits of the proposed method, a comparison with a common traditional method was made. The most common modern method for capturing foot geometry consists of the patient sitting in a chair and gently resting their foot on a flat glass plate as the plantar foot geometry is scanned from below. Contact with the flat glass plate conveniently adjusts the foot's posture so that it is suitable for FO design. Unlike the proposed method, however, the extent to which posture is adjusted is inexact and distorts the plantar soft tissues through contact with the glass. As will be seen in the next section, this 


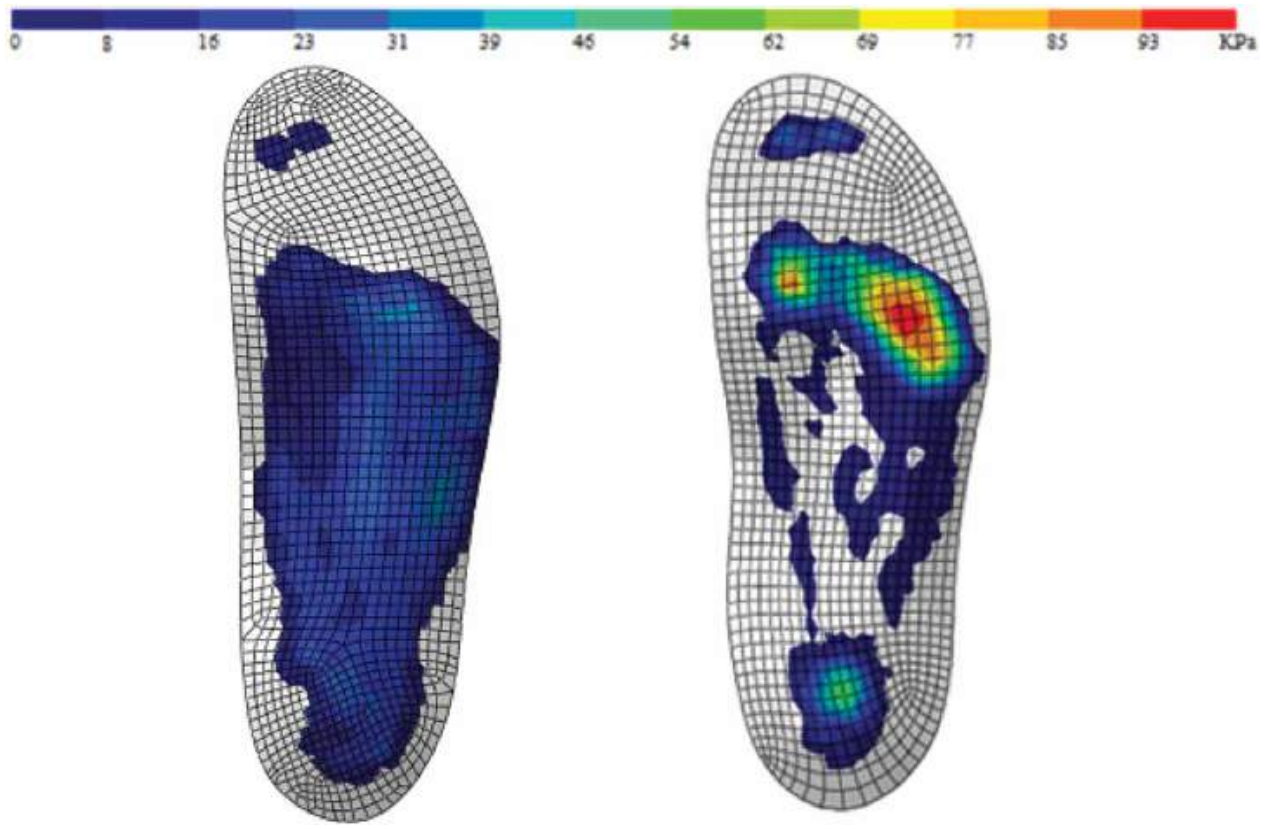

Fig. 9: Comparison of simulated pressure distributions between the proposed method and a conventional method.

adversely affects the results. Once the foot geometry has been captured, CAD software can then be used to design the FO about the plantar foot geometry.

Rather than carry out the flat glass plate method physically, it was done computationally to both expedite the test and ensure similar alignment with the proposed method. In the flat glass plate simulation, the subject's foot was loaded onto flat ground (the glass plate) until the heel just touched the ground surface. The deformed foot surface geometry was exported and a FO was designed about the plantar surface using the FO design program developed by Lochner et al. [16].

The subject's foot model was then loaded on to each the FO from the flat glass plate method and the FO from the proposed method. Finally, the resulting plantar pressures distributions were exported from Abaqus and compared.

\section{RESULTS AND DISCUSSION}

The proposed method yielded a $61 \%$ reduction in peak plantar pressure when compared to the simulated conventional method (Fig. 9). For the simulated conventional method, the compression of the plantar soft tissues caused by contact with ground resulted in a FO design with poorer conformity to the undeformed plantar foot geometry than the proposed method, and consequently, higher peak plantar pressures. The difference is more apparent in the forefoot where greater tissue deformation resulted from contact with ground. Furthermore, the lack of an optimization routine did not provide the opportunity to adjust the FO design in high pressure areas.
Other conventional modern and traditional methods may distort plantar soft tissue to a lesser extent. For example, traditional casting methods build the negative plaster cast about the patient's non weight bearing foot. However, the plaster does have some effect on the soft tissues it is in contact with, the practitioner affects the soft tissue in the process of correcting posture, and some accuracy is lost during the creation of a positive cast due to problems with plaster expansion. The proposed method is the only known method that does not apply pressure to the plantar foot surface that allows for realistic control over the foot's posture. Furthermore, it is the only method that allows for the visualization of pressure distribution and the opportunity for optimization prior to fabrication of the FO.

The usefulness of the proposed solution is highly dependent on the accuracy of the simulation model. The practitioner prescribes recommendations, but it is the model that carries them out. For these reasons, FEA was selected as the simulation technique given its wide acceptance and validation in foot modeling. Its main deficiency is the amount of computing power it requires. Thus, alternative simulation methods should be considered. The procedure was further time consuming due to the considerable amount of skilled computer labor required as models were passed between CAD and FEA and settings were adjusted. However, in contrast to conventional methods, this labour has the potential to be fully automated with minimal subjective input.

Though not tested here, other metrics would also benefit from the proposed methods. For example, stress in the plantar fascia could be minimized 
through incremental variations in FO design. Other potential metrics include stresses in the soft tissues, positions of specific joints, and stresses in bones. Given the adaptation of the model to dynamic analysis, other metrics including joint articulation velocities could be considered. The example optimization routine in the current work was carried out manually. An area for continuing research is the automation of the optimization routines.

\section{SUMMARY}

The current work incorporates simulation methods into the FO development process via two additional steps. The proposed method reduces the subjective input required by the clinic practitioner and lab operator, allows for the fully non weight bearing passive digitization of the foot, isolates all posture adjustment to a single simulation step, and allows for design optimization prior to fabrication. It was shown that a FO developed with the proposed method yielded lower plantar pressures than for a FO developed with a conventional method. This was due to the proposed method's ability to control posture without distorting the plantar soft tissues and the opportunity provided to optimize a FOs' ability to redistribute plantar pressures. Though the proposed method employed the postural control paradigms devised by Root et al., it is not limited to these. Further work includes the automation of optimization routines (via algorithms with closed loop feedback control) for a variety of metrics and adaptation towards dynamic analysis. Other applications of the proposed methods include the development processes for orthoses and prostheses for other parts of the body.

\section{REFERENCES}

[1] Arakawa, M.; Miyake, S.; Nakao, S.: Approximate multi-objective optimization of medical foot support: Case of 3D shape optimization, American Society of Mechanical Engineers International Design Engineering Technical Conferences, 2009.

[2] Cheung, J.; Zhang, M.: Parametric design of pressure-relieving foot orthosis using statisticsbased finite element method, Medical Engineering Physics, 30, 2008, 269-277.

[3] Crabtree, P.; Dhokia, V.; Newman, S.; Ansell, M.: Manufacturing methodology for personalised symptom-specific sports insoles, Robotics Computer Integration Manufacturing, 25, 2009, 972-979.

[4] Erdemir, A.; Saucerman, J.; Lemmon, D.; Loppnow, B.; Turso, B.; Ulbrecht, J.; Cavanagh, P.: Local plantar pressure relief in therapeutic footwear: design guidelines from finite element models, Journal Biomechanics, 38, 2005.
[5] Foort, J.; Spiers, R.; Bannon, M.: Experimental fittings of sockets for below-knee amputees using computer aided design and manufacturing techniques, Prosthetics Orthotics International, 9, 1985, 46-47.

[6] Goske, S.; Erdemir, A.; Petre, M.; Budhabhatti, S.; Cavanagh P.: Reduction of plantar heel pressures: Insole design using finite element analysis, Journal Biomechanics, 39, 2006, 2363-2370.

[7] Gu, Y.; Li, S.; Lake, M.; Zeng, Y.; Ren, X.; Li, Z.: Image-based midsole insert design and the material effects on heel plantar pressure distribution during simulated walking loads, Computer Method Biomechanics Biomedical Engineering, 14, 2011, 747-753.

[8] Houle, P.; Beaulieu, E.; Liu, Z; Novel fully integrated computer system for custom footwear: From 3D digitization to manufacturing, Electronic Imaging, 2010.

[9] Hsu, Y.; Gung, Y.; Shih, S.; Feng, C.; Wei, S.; Yu, C.; Chen, C.: Using an Optimization Approach to Design an Insole for Lowering Plantar Fascia Stress-A Finite Element Study, Annals Biomedical Engineering, 36, 2008.

[10] Hussein, S.: An intelligent design for foot orthoses, Key Eng Mat, 2008, 381-382.

[11] Jones, D.; Impact of advanced manufacturing technology on prosthetic and orthotic practice, Journal of Biomedical Engineering 10, 1988.

[12] Kelaher, D.; Mirka, G.; Dudziak, K.: Effects of semi-rigid arch-support orthotics: an investigation with potential ergonomic implications, Applied Ergonomics, 31, 2000.

[13] Klasson, B.: Computer aided design, computer aided manufacture and other computer aids in prosthetics and orthotics, Prosthetics Orthotics International, 9, 1985, 3-11.

[14] Kosolapov, S.: Robust Algorithms Sequence for Structured Light 3D Scanner Adapted for Human Foot 3D Imaging, Journal of Communications and Computer, 8, 2011, 595-598.

[15] Lee, W.; Lee, C.; Leung, A.; Hutchins, S.: Is it important to position foot in subtalar joint neutral position during non-weightbearing molding for foot orthoses?, Journal of Rehabilitation Research, 49, 2012, 459-66.

[16] Lochner, S.; Huissoon, J.; Bedi, S.: Development of a patient-specific anatomical foot model from structured light scan data, Computer Methods Biomechanics Biomedical Engineering, 2012.

[17] Lochner, S.; Huissoon, J.; Bedi, S.: Parametric design of Custom foot orthotic model. Computer-Aided Design and Applications, 9, 2012, 1-11.

[18] Lochner, S.: Computer Aided Engineering in the Foot Orthosis Development Process, 2013.

[19] Pallari, J.; Dalgarno, K.; Woodburn, J.: Mass customization of foot orthoses for rheumatoid 
arthritis using selective laser sintering, IEEE Transactions on Biomedical Engineering, 57, 2010, 1371-1378.

[20] Philps, J.: The Functional Foot Orthosis, Edinburgh: Churchill Livingstone, 1995.

[21] Rodriguez, J.: Computer vision of the foot sole based on laser metrology and algorithms of artificial intelligence, Optical Engineering, 48, 2009.

[22] Root, M.; Weed, J.; Orien, W.: Neutral Position Casting Techniques, Clinical Biomechanics Corporation, 1971.

[23] Sobel, E.; Levitz, S.: Reappraisal of the negative impression cast and the subtalar joint neutral position, Journal American Podiatric Medical Association, 87, 1997, 32-33.

[24] Telfer, S.; Pallari J.; Munguia J.; Dalgarno K.; McGeough M.; Woodburn J.: Embracing additive manufacture: implications for foot and ankle orthosis design, BMC Musculseletal Disorders, 13, 2012.

[25] Trinidad, L.: Engineering modeling, analysis and optimal design of custom foot orthotic, ProQues. Disser. and Theses, 2011.

[26] Tsung, B.; Zhang, M.; Mak, A.; Wong, M.: Effectiveness of insoles on plantar pressure redistribution, Journal of rehabilitation research and development, 2004. 